\title{
Apnea in Neonates - Concepts and Controversies
}

\author{
Santosh Kumar K* \\ Department of Neonatology, Saveetha medical college and hospital, India
}

*Corresponding author: Santosh Kumar K, Department of Neonatology, Saveetha medical college and hospital, India

\begin{abstract}
Apnoea of prematurity (AOP) is a common problem affecting premature infants. Apnoea is defined as cessation of breathing for more than 20 seconds or for lesser duration when associated with bradycardia and desaturation. The most likely and accepted pathogenesis is the "physiologic" immaturity of respiratory control in the neonates. The physiological immaturities include altered ventilatory responses to hypoxia, hypercapnia, and altered sleep states. Other hypotheses include gastroesophageal reflux and anaemia which are still controversial. Three types have been identified namely obstructive, central and mixed types of apnoea. Management options include the use of supplemental oxygen, position chages and drug therapy with methyxanthines. Other supportive therapies include kangaroo care, packed cell transfusions. The long term neurodevelopmental consequences of AOP and its treatment still needs to be studied further..
\end{abstract}

Keywords: Apnoea of Prematurity; Premature Infant; Neurodevelopment; Methylxanthine Therapy

\section{Introduction}

Prematurity is associated with problems of temperature regulation, acquisition of oral feeding skills, and immature control of respiration leading to apnoea [1]. Resolution of apnoea and establishment of a normal respiratory pattern is a major developmental milestone for premature infants. The accepted definition of apnoea of prematurity (AOP) defines it as cessation of breathing for more than 15-20 seconds, or accompanied by oxygen desaturation $\left(\mathrm{SpO}_{2} \leq 80 \%\right.$ for $\geq 4$ seconds) and bradycardia (heart rate<100) [2]. Pathogenesis AOP is linked to the developmental immaturity of the respiratory control of breathing and it resolves on its own over period of time. AOP reflects a "physiological" rather than a "pathological" immature state of respiratory control.

\section{Incidence}

The incidence of AOP is inversely correlated with gestational age and birth weight. Seven percent of neonates born at 34 to 35 weeks gestation, $15 \%$ at 32 to 33 weeks, $54 \%$ at 30 to 31 weeks and nearly all infants born at 28 weeks develop apnoea. Apnoea is classified into three subtypes: central, obstructive, or mixed. Central apnoea accounts for approximately $10 \%$ to $25 \%$ of all cases of apnoea, with obstructive apnoea accounting for $10 \%$ to $25 \%$ and mixed for $50 \%$ to $75 \%[3,4]$.

\section{Fetal to Neonatal Transition}

The fetus moves from an oxygen-poor hypoxic environment in utero where the $\mathrm{PaO}_{2}$ is $25-27 \mathrm{mmHg}$, to an oxygen-rich environment after birth. The postnatal rise in $\mathrm{PaO}_{2}$ effectively reduces the sensitivity of the peripheral chemo receptors, resulting in delayed onset of spontaneous respiratory efforts when neonates are exposed to $100 \%$ oxygen during postnatal resuscitation. Hence the neonates need adapt to the postnatal environment by altering the respiratory and ventilator controls. But because of the immature respiratory control pattern and chemoreceptor function in premature infants there is a delay in this adjustment [5].

\section{Ventilatory Response to Hypoxia}

The ventilatory response to hypoxia after birth in premature infants elicits an initial transient increase in respiratory rate and tidal volume that lasts for 1-2 min, followed by a late, sustained decline in spontaneous breathing that may last for several weeks. This late decline in spontaneous breathing is termed hypoxic ventilatory depression, which could be associated with the delayed postnatal respiratory adjustment [6]. Peripheral chemoreceptor stimulation may also lead to apnoea secondary to hypocapnia seen after hyperventilation. The apnoeic threshold of $\mathrm{CO}_{2}$ secondary to 
hyperventilation along with peripheral chemoreceptor activation in response to hyperventilation may lead to apnoea [7].

\section{Ventilatory Response to Hypercapnia}

Preterm infants increase ventilation by prolonging the period of expiration, but not increasing breath frequency or overall tidal volume, leading to less minute ventilation in response to hypercapnea. This poor hypercapnic ventilatory response is more pronounced in premature infants with apnoea than without apnoea [8]. Contradictory movements of respiratory muscles in response to hypercapnia may also play a role in AOP.

\section{Ventilatory Responses to Laryngeal Chemoreflex}

Activation of the laryngeal mucosa in premature infants can lead to apnea, bradycardia, and hypotension. While this response is assumed to be a protective reflex, an exaggerated response may cause AOP. This reflex-induced apnea is termed the laryngeal chemoreflex and is mediated through superior laryngeal nerve afferents [9].

\section{Neurotransmitters and Apnoea}

Enhanced sensitivity to inhibitory neurotransmitters, such as gamma-aminobutyric acid (GABA), adenosine has been postulated as a pathogenic mechanism. GABA is the major inhibitory neurotransmitter in the CNS. Adenosine is a product of adenosine triphosphate and is formed as a consequence of metabolic and neural activity in the brain, especially during hypoxia. Reports have found an interaction between adenosine and GABA in the regulation of breathing. This association is further strengthened by the observations that adenosine receptors are expressed in GABAcontaining neurons. The binding of adenosine to its receptor may be involved with the release of GABA and thus inhibit respiration leading to apnoea [10].

\section{Genetic Variability and Apnoea}

Tamim et al. [11] reported a higher proportion of first degree mating for infants with AOP compared with those without AOP. Further need to be done in this area to give a clear conclusion regarding the genetic factors role in AOP.

\section{Effects of Sleep State and Movements on Apnoea}

Premature infants spend a large proportion of their time in rapid eye movement (REM) sleep, with a relatively smaller amount in wakefulness. During REM sleep, these infants have more paradoxical breathing with a less stable baseline of oxygen saturation. Therefore, apnoea occurs more frequently in REM sleep than in quiet sleep12. Arousal from REM sleep appears to be a precursor to apnoea associated with oxygen desaturation in premature infants since motor activities after arousal are typically associated with laryngeal closure. Therefore, movements frequently precede or occur simultaneously with apnoea, and arousal from sleep may cause the apnoea rather than terminate it $[12,13]$.

\section{Other Factors Involved in Apnoea}

While immature respiratory control is the primary cause of apnoea in the premature infant, many coexisting factors can potentiate or worsen apnoea. Apnoea is a common presenting sign of both local and systemic infection13. Apnoea can be secondary to central nervous system diseases like intracranial haemorrhage, hypoxic-ischemic encephalopathy, and seizures. Exposure to cooler temperatures decreased the duration and frequency of AOP, while elevated body temperature increased the incidence of AOP, suggesting that apnoea is related to metabolic state and environmental temperature [1,2]. Other factors that have been associated with apnoea in premature infants include glucose or electrolyte imbalance, presence of a hemodynamically significant patent ductus arteriosus (hs-PDA) [1,2]. Anaemia is also associated with apnoea because of lowered oxygen-carrying capacity of red blood cells that leads to hypoxia, resulting in respiratory depression. Drugs including narcotic analgesics and magnesium sulfate, can lead to apnea in infants. The role of Gastroesophageal reflux in causing apnoea is controversial also, there is no evidence to support the use of anti-reflux medications for the prevention of aponea [14].

\section{Interventions for Premature Infants with Apnoea}

Interventions for AOP include efforts to reduce work of breathing and/or increase respiratory efforts.

\section{Non Pharmacological Therapies}

Prone Position

Prone positioning can improve thoracoabdominal synchrony and stabilize the chest wall without affecting breathing pattern or $\mathrm{SpO}_{2}$. Several studies have demonstrated that prone position reduces AOP [15]. Extension of the neck $15^{\circ}$ from the prone position is referred to as the head -tilt position, which has been found to decrease episodes of oxygen desaturation and it should be considered as a first-line intervention in infants with AOP However, head- tilt position has not been shown to work in combination with pharmacologic therapy.

\section{Continuous Positive Airway Pressure and Nasal Intermittent Positive Pressure Ventilation}

CPAP at 4-6 $\mathrm{cm} \mathrm{H}_{2} \mathrm{O}$ has proven a safe and effective therapy for AOP. CPAP delivers a continuous distending pressure via the infant's pharynx to the airways to prevent both pharyngeal collapse and alveolar atelectasis [16]. Therefore, CPAP can enhance functional residual capacity and reduce the work of breathing, improving oxygenation and decreasing bradycardia. CPAP works effectively to reduce the incidence of obstruction, but it has no clear efficacy in central AOP. An extension of CPAP is the administration of NIPPV which has been shown to be more effective than CPAP in preventing extubating failure and for the treatment of AOP [17].

\section{Other Therapies}

\section{KMC}

Maternal kangaroo care, also known as skin-to-skin care for premature infants, has calming effects on the baby's clinical status and vital signs. It has shown to be effective in reducing the apnoea $[18,19]$. 


\section{Sensory Stimulation}

Several studies suggest that sensory stimulants, including tactile and olfactory stimulation, are useful in the treatment or prevention of AOP. Tactile stimulation is the most common intervention in response to AOP. This simple intervention most likely works by generating excitatory, nonspecific neuronal activity in the brainstem centre to stimulate respiratory activity. However, cutaneous stimulation often arouses the infant and markedly affects breathing pattern in premature infants. Systematic review has shown that kinesthetic stimulation is not effective at preventing AOP [20]. Olfactory stimulation has also been used for the treatment of AOP. Pleasant odors elicited increased respiratory drive, whereas unpleasant odors caused decreased respiratory effort, during active sleep when apnoea is more common. Vanillin, a stimulus known to affect the olfactory nerve, was used to treat refractory apnoea and bradycardia unresponsive to both caffeine. Studies have concluded that the presence of a pleasant odour helped the infants to better regulate their respiratory patterns [21]. $\mathrm{CO}_{2}$ inhalation, $\mathrm{CO}_{2}$ is the physiologic stimulus for breathing in mammals. Apnoea occurs when the $\mathrm{CO}_{2}$ baseline decreases below the apnoea threshold. A rise in $\mathrm{CO}_{2}$ of 1 to $2 \mathrm{mmHg}$ above the apnoea threshold will reduce or abolish apnoea. Recently, a randomized controlled trial of theophylline versus $\mathrm{CO}_{2}$ inhalation for treating AOP showed that inhalation of a low $\mathrm{CO}_{2}$ concentration $(0.8 \%)$ in premature infants is as effective as theophylline in decreasing apnoea. This exposure to $0.8 \% \mathrm{CO}_{2}$ also had no effect on cerebral blood flow velocity. The authors concluded that $\mathrm{CO}_{2}$ may be a better treatment for AOP than methylxanthines. However, it is likely that infants will quickly accommodate to an inspiratory $\mathrm{CO}_{2}$ concentration, and the effectiveness of long-term exposure is not known [22]. An increase in upper airway resistance may also play a significant role in AOP. Nasogastric tubes have been documented to increase nasal airway resistance by $50 \%$. Therefore, orogastric feeding tubes are sometimes preferred in premature infants with apnoeic events. Interestingly, transpyloric feedings, especially when limited to human milk, have recently been shown to be safe and reduce episodes of apnoea and bradycardia in premature infants with suspected gastroesophageal reflux [23].

\section{Thermoneutral Range}

A mild increase in body temperature in infants enhances the instability of the breathing pattern. However, a specific environmental temperature to reduce the incidence or severity of AOP is not known, and more research is required.

\section{Packed Cell Transfusion}

Anemia can lead to AOP, and a proposed mechanism to treat AOP is transfusion of red blood cells to increase oxygen carrying capacity. However, data on the effect of blood transfusion on AOP is not clear. Because of the lack of clear cut evidence transfusion to treat AOP may not be recommended as a routine unless the neonate fulfils the recommened transfusion criteria for preterm neonates [24].

\section{Methylxanthine Therapy}

Methylxanthine compounds such as caffeine, theophylline, and aminophylline have been administered to premature infants as respiratory stimulants to decrease AOP. These drugs are powerful central nervous system stimulants and are likely to reduce apnea by multiple physiological and pharmacological mechanisms [25,26].

These drugs are antagonist of adenosine receptor and work by increasing the minute ventilation, improving the $\mathrm{CO}_{2}$ sensitivity and neural respiratory drive and decreasing the hypoxic respiratory depression. Other mechanisms include improved diaphragmatic contraction and improved respiratory muscle function [25]. Systematic reviews of caffeine therapy in AOP have shown that both caffeine and theophylline are effective in reducing apnoea. Caffeine is the preferred of the two drugs considering its wider therapeutic range and the plasma half-life making it ideal for once a day administration. Further studies have shown that the incidence bronchopulmonary dysplasia and neurodevelopment disabilities have decreased with the use of Caffeine. The potential mechanism of neuroprotection is not clearly understood but could be explained by the decrease of ventilator-induced lung injury (VILI) due to the use of caffeine [26-28]. Caffeine is usually available as caffeine citrate and the active component comprises of only $50 \%$ of the total dose. Caffine is generally given as a loading dose of $10 \mathrm{mg} / \mathrm{kg}$ caffeine (i.v. or orally) followed by a maintenance dose of $5 \mathrm{mg} / \mathrm{kg}$ once daily. Higher doses have been studied but have shown to be of little use except in cases of refractory AOP $[27,28]$.

Theophylline is usually recommended at a loading dose of 5-6 $\mathrm{mg} / \mathrm{kg}$, followed by maintenance doses of $2-6 \mathrm{mg} / \mathrm{kg} /$ day in divided into two or three daily doses. Therapy with methylxanthines is associated with few adverse events. Toxic levels may produce tachycardia, cardiac dysrhythmias, and feeding intolerance or, at very high doses, may precipitate seizures. Mild diuresis and delayed gastric emptying can also be seen in very low birth weight infants on methyxanthine therapy. Methylxanthines also increase energy expenditure, possibly leading to diminished growth in premature infants hence a extra caloric requirement is necessary in infants treated with theophylline [26-28]. Doxapram is a potent respiratory stimulant previously used for the management of apnoea refractory to methylxanthine therapy. The use of doxapram is controversial because of its reported adverse effects with include irritability, elevated blood pressure, and gastric retention, increase in cerebral oxygen consumption and a decrease in oxygen delivery. This is probably mediated by a decrease of cerebral blood flow. Therefore, doxapram is not routinely recommended for AOP since its side effects and long-term benefits versus potential harm are concerning [29]. Drugs are generally stopped when the neonate is apnoea free for 7 days or has reached 34 weeks Corrected gestational age and it is generally advisable to stop the drugs 4 to 5 days prior to discharge of these neonates as the drugs have long half-life and premature discharge after stopping the drugs may lead to dire consequences. 


\section{Consequences of AOP}

It is difficult to prove a link between apnoea and poor neurodevelopment outcomes due to the possible coexistence of neurological injury in premature infants. In early studies, no differencesinneurodevelopmentoutcomeswerefound between AOP and control infants [30,31]. Janvier et al30. found that an increased number of AOP days were associated with neurodevelopment impairment such as cerebral palsy and blindness at 3 years of age. Recently, researchers found that a higher frequency and severity of AOP were associated with a higher incidence of unfavorable outcomes or death. Possible explanation for this is that multiple recurrent hypoxic and bradycardia following AOP may cause longterm cerebral dysfunction. Apnoea is a very common symptom in premature infants. Treatment options include pharmacological and physical and sensory stimulation. The long-term consequences of severe, recurrent apnoea-associated bradycardia and desaturation still needs to be studied in detail in future studies.

\section{References}

1. Stokowski LA (2005) A primer on Apnea of prematurity. Adv Neonatal Care 5(3): 155-170.

2. Moriette G, Lescure S, El Ayoubi M, Lopez E (2010) Apnea of prematurity: what"s new? Arch Pediatr 17(2): 186-190.

3. Martin RJ, Abu Shaweesh JM, Baird TM (2004) Apnoea of prematurity. Paediatr Respir Rev 5(Suppl 1): S377-S382

4. Mercer JS, Erickson Owens DA, Graves B, Haley MM (2007) Evidencebased practices for the fetal to newborn transition. J Midwifery Womens Health 52(3) :262-272.

5. Darnall RA, Ariagno RL, Kinney HC (2006) The late preterm infant and the control of breathing, sleep, and brainstem development: a review. Clin Perinatol 33(4): 883-914.

6. Nock ML, Difiore JM, Arko K, Martin RJ (2004) Relationship of the ventilatory response to hypoxia with neonatal apnea in preterm infants. J Pediatr 144(3): 291-295.

7. Khan A, Qurashi M, Kwiatkowski K, Cates D, Rigatto H (2005) Measurement of the $\mathrm{CO} 2$ apneic threshold in newborn infants: possible relevance for periodic breathing and apnea. J Appl Physiol 98(4): 11711176.

8. Darnall RA (2010) The role of $\mathrm{CO}_{2}$ and central chemoreception in the control of breathing in the fetus and the neonate. Respir Physiol Neurobiol 173(3): 201-212.

9. Thach BT (2007) Maturation of cough and other reflexes that protect the fetal and neonatal airway. Pulm Pharmacol Ther 20 (4): 365-370.

10. Simakajornboon N, Kuptanon $\mathrm{T}$ (2005) Maturational changes in neuromodulation of central pathways underlying hypoxic ventilatory response. Respir Physiol Neurobiol 149(1-3): 273-286.

11. Tamim H, Khogali M, Beydoun H, Melki I, Yunis K, et al. (2003) Consanguinity and apnea of prematurity. Am J Epidemiol 158(10): 942946.

12. Lehtonen L, Martin RJ (2004) Ontogeny of sleep and awake states in relation to breathing in preterm infants. Semin Neonatol 9 (3): 229-238
13. Kamaluddeen M, Lodha A, Akierman A (2009) Non-Rotavirus infection causing apnea in a neonate. Indian J Pediatr 76 (10): 1051-1052.

14. Peter CS, Sprodowski N, Bohnhorst B (2002) Gastroesophageal reflux and apnea of prematurity: No temporal relationship. Pediatrics 109(1): 8-11.

15. Hannam S, Pressler R, Rafferty GF, Peacock JL, Greenough A, et al. (2006) Effect of prone and supine position on sleep, apneas, and arousal in preterm infants. Pediatrics 118(1): 101-107.

16. Millar D, Kirpalani H (2004) Benefits of noninvasive ventilation. Indian Pediatr 41(10): 1008-1017.

17. Lemyre B, Davis PG, De Paoli AG (2002) Nasal intermittent positive pressure ventilation (NIPPV) versus nasal continuous positive airway pressure (NCPAP) for apnea of prematurity. Cochrane Database Syst.

18. Gathwala G, Singh B, Singh J (2010) Effect of Kangaroo Mother Care on physical growth, breastfeeding and its acceptability. Trop Doct 40(4): 199-202

19. Heimann K1, Vaessen P, Peschgens T, Stanzel S, Wenzl TG, et al. (2010) Impact of skin to skin care, prone and supine positioning on cardiorespiratory parameters and hermoregulation in premature infants. Neonatology 97(4): 311-317 34.

20. Gaugler C, Marlier L, Messer J (2007) Sensory stimulations for the treatment of idiopathic apneas of prematurity. Arch Pediatr 14 (5): 485489.

21. Marlier L, Gaugler C, Messer J (2005) Olfactory stimulation prevents apnea in premature newborns. Pediatrics 115(1): 83-88.

22. Joseph LJ, Goldberg S, Picard E (2009) CO2 treatment for apnea. J Pediatr 154(4): 627-628.

23. Bohnhorst B, Cech K, Peter C, Doerdelmann M (2010) Oral versus nasal route for placing feeding tubes: no effect on hypoxemia and bradycardia in infants with apnea of prematurity. Neonatology 98(2): 143-149.

24. Valieva OA, Strandjord TP, Mayock DE, Juul SE (2009) Effects of transfusions in extremely low birth weight infants: a retrospective study. J Pediatr 155(3): 331-337.

25. Aranda JV1, Beharry K, Valencia GB, Natarajan G, Davis J (2010) Caffeine impact on neonatal morbidities. J Matern Fetal Neonatal Med 23(Suppl 3): $20-23$.

26. Henderson Smart DJ, Steer PA (2010) Caffeine versus theophylline for apnea in preterm infants. Cochrane Database Syst Rev 20 (1): CD000273.

27. Schmidt B, Roberts RS, Davis P et al. (2006) Caffeine therapy for apnea of prematurity. N Engl J Med 354(20): 2112-2121.

28. Schmidt B, Roberts RS, Davis P, Doyle LW, Barrington KJ, et al. (2007) Long-term effects of caffeine therapy for apnea of prematurity. N Engl J Med 357 (19): 1893-1902.

29. Yost CS (2006) A new look at the respiratory stimulant doxapram. CNS Drug Rev 12(3-4): 236-249.

30. Mojica N, Jadeja N, Ostfeld B, Hiatt M, Hegyi T, et al. (1993) Neurodevelopmental outcome of infants with apnea of infancy. Am J Perinatol 10 (3): 208-211.

31. Hermann C, Keller T, von Gontard A, Kribs A, Roth B, et al. (2007) Factors influencing apnea and bradycardia of prematurity-mplications for neurodevelopment. Neonatology 91(3): 155-161. 


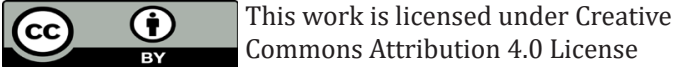

To Submit Your Article Click Here: Submit Article

DOI: $10.32474 /$ PAPN.2019.02.000134

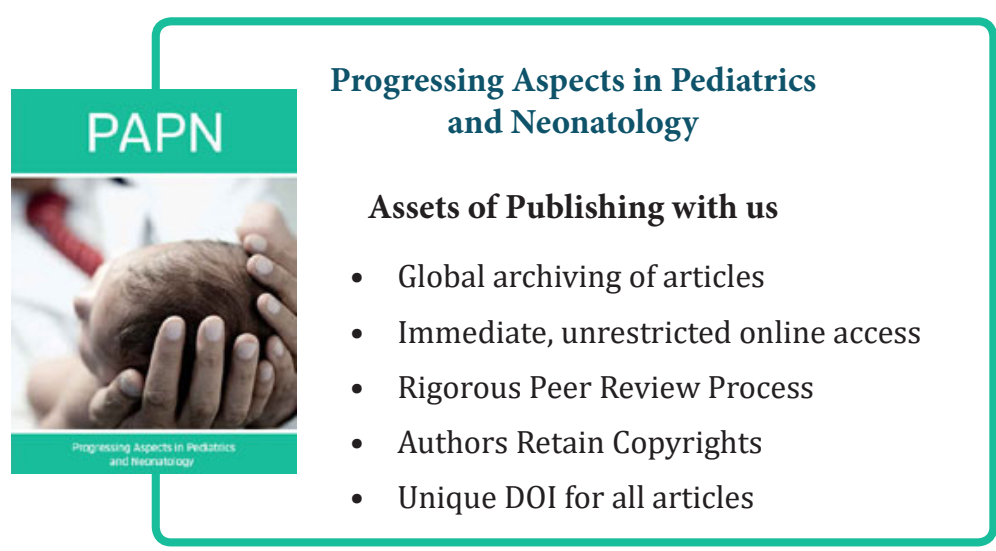

